\title{
Autoestima entre homens e mulheres praticantes de musculação
}

\author{
Self esteem between men and women practitioner of bodybuilding \\ Autoestima entre hombres y mujeres practicantes el culturismo
}

Recebido: 24/08/2021 | Revisado: 03/09/2021 | Aceito: 03/09/2021 | Publicado: 06/09/2021

\author{
Moisés Augusto de Oliveira Borges \\ ORCID: https://orcid.org/0000-0003-3372-5932 \\ Universidade Federal Rural do Rio de Janeiro, Brasil \\ E-mail: m.oliveiraborges@hotmail.com \\ Gabriela Simões \\ ORCID: https://orcid.org/0000-0003-1041-340X \\ Universidade Federal Rural do Rio de Janeiro, Brasil \\ E-mail: gabssimoes9@gmail.com \\ Nayani Vitória Ribeiro Albuquerque \\ ORCID: https://orcid.org/0000-0002-7342-7094 \\ Universidade Federal Rural do Rio de Janeiro, Brasil \\ E-mail: nayanevitoria7@ hotmail.com \\ Lorena Rodrigues Vieira Mendes \\ ORCID: https://orcid.org/0000-0003-1866-8263 \\ Universidade Federal Rural do Rio de Janeiro, Brasil \\ E-mail: lorenamendes38@gmail.com \\ Leonardo da Silva Santos \\ ORCID: https://orcid.org/0000-0003-0401-9182 \\ Universidade Federal Rural do Rio de Janeiro, Brasil \\ E-mail: leoufrrj2019@gmail.com \\ Rafael dos Santos Pereira \\ ORCID: https://orcid.org/0000-0002-8026-5986 \\ Universidade Federal do Rio de Janeiro, Brasil \\ E-mail: rsp544@gmail.com \\ Maria de Lourdes Augusta de Oliveira \\ ORCID: https://orcid.org/0000-0003-0147-3371 \\ Prefeitura da Cidade do Rio de Janeiro, Brasil \\ E-mail: mlou.ped@gmail.com \\ Thiago de Sousa Rosa \\ ORCID: https://orcid.org/0000-0003-4774-3322 \\ Universidade Federal Rural do Rio de Janeiro, Brasil \\ E-mail: thiago.rosaef@gmail.com \\ Ricardo Ruffoni \\ ORCID: https://orcid.org/0000-0001-5954-5740 \\ Universidade Federal Rural do Rio de Janeiro, Brasil \\ E-mail: prof.ruffoni@gmail.com
}

\begin{abstract}
Resumo
Objetivo: Esse estudo teve por objetivo avaliar e comparar os níveis de autoestima de praticantes masculinos e femininos de musculação. Metodologia: Adotou-se uma abordagem quantitativa, de delineamento transversal e de caráter descritivo e comparativo. Compuseram a amostra 120 praticantes de musculação, sendo 60 do sexo masculino e 60 do sexo feminino, com idade média de 25 anos em ambos os grupos. Para a coleta de dados recorreu-se a Escala de Autoestima de Rosenberg. Os dados foram tratados estatisticamente no software SPSS 20.0, de forma descritiva e

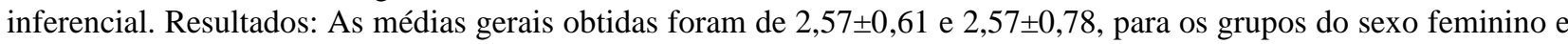
masculino, respectivamente. Os praticantes indicaram autoestima classificada como média. As comparações não indicaram diferença com significância estatística. Considerações finais: Os homens e mulheres praticantes de musculação investigados, com tempo de prática equivalentes, possuem autoestimas semelhantes. Dessarte, é possível supor que a prática regular de exercício físico, nesse caso a musculação, pode contribuir para elevar os níveis de autoestima do indivíduo.

Palavras-chave: Autoconfiança; Atividade física regular; Exercício físico; Qualidade de vida; Escala de autoestima de Rosenberg.
\end{abstract}

\footnotetext{
Abstract

Objective: This study aimed to evaluate and compare the levels of self-esteem of male and female bodybuilders. Methodology: A quantitative, cross-sectional and descriptive and comparative approach was adopted. The sample comprised 120 bodybuilding practitioners, 60 male and 60 female, with an average age of 25 years in both groups.
} 
Rosenberg's Self-Esteem Scale was used for data collection. The data were treated statistically in the software SPSS

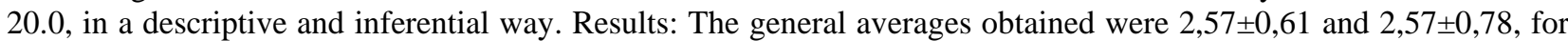
the female and male groups, respectively. The practitioners indicated self-esteem classified as average. Comparisons did not indicate any statistically significant difference. Final considerations: The men and women investigated for bodybuilding investigated, with equivalent practice time, have similar self-esteem. Thus, it is possible to assume that the regular practice of physical exercise, in this case, weight training, can contribute to raise the individual's selfesteem levels.

Keywords: Self confidence; Regular physical activity; Physical exercise; Quality of life; Rosenberg Self-esteem Scale.

\section{Resumen}

Objetivo: Este estudio tuvo como objetivo evaluar y comparar los niveles de autoestima de culturistas masculinos y femeninos. Metodología: Se adoptó un enfoque cuantitativo, transversal, descriptivo y comparativo. La muestra estuvo formada por 120 culturistas, 60 hombres y 60 mujeres, con una edad promedio de 25 años en ambos grupos. Para la recolección de datos se utilizó la Escala de Autoestima de Rosenberg. Los datos se trataron estadísticamente en el software SPSS 20.0, de forma descriptiva e inferencial. Resultados: Los promedios generales obtenidos fueron $2,57 \pm 0,61$ y 2,57 $\pm 0,78$ para los grupos de mujeres y hombres, respectivamente. Los practicantes indicaron una autoestima clasificada como promedio. Las comparaciones no indicaron una diferencia estadísticamente significativa. Consideraciones finales: Los hombres y mujeres practicantes de culturismo investigados, con tiempo de práctica equivalente, tienen una autoestima similar. Así, es posible asumir que la práctica regular de ejercicio físico, en este caso el entrenamiento con pesas, puede contribuir a elevar los niveles de autoestima del individuo.

Palabras clave: Autoconfianza; Actividad física regular; Ejercicio físico; Calidad de vida; Escala de autoestima de Rosenberg.

\section{Introdução}

Existem diferentes abordagens teóricas e, consequentemente, distintas expressões adotadas para conceituar a autoestima. De maneira geral, a autoestima se refere a uma atribuição de valor intrínseca do indivíduo sobre si nos acontecimentos da vida, a qual envolve valores positivos ou negativos. Enquanto constructo complexo, cabe mencionar que a autoestima não é estável, ou seja, é passível de variações mediante, por exemplo, experiências agradáveis ou desagradáveis na vida do indivíduo. Ainda, esta envolve valores, crenças e atitudes, assim como é influenciada pelo contexto cultural e social que circundam o indivíduo (Schultheisz \& Aprile, 2013).

Dessa forma, a autoestima envolve uma avaliação subjetiva, sendo definida como sentimento, apreço e consideração que uma pessoa tem por si própria (Morales \& González, 2014). Cabe destacar que é possível, por exemplo, que um indivíduo tenha boa autoestima com relação ao trabalho e, ao mesmo tempo, baixa autoestima no que diz respeito à capacidade física (Salmivalli et al., 1999). Nesse contexto, indivíduos satisfeitos com os acontecimentos da vida tendem a se sentirem competentes e confiantes, o que influenciará em sua valoração pessoal (Schultheisz \& Aprile, 2013). Por isso, autores indicam que um indivíduo tende a ter autoestima elevada em relação as práticas que se sente competente, pois, na maioria das vezes, ele as domina. Diferente disso, a autoestima será baixa nas atividades que não forem de seu domínio (Oliveira, 2001; Gobitta \& Guzzo, 2002).

Enquanto característica da subjetividade humana, a autoestima apresenta um papel importante na forma como uma pessoa se relacionada consigo mesma e como estabelece relações com os pares, além de relacionar-se com a construção de sua identidade (Vasconcelos, 2017). Ainda, Schultheisz e Aprile (2013) apontam que a autoestima é influenciada por experiências da infância e pelo ambiente em que a pessoa viveu, assim como as características de sua personalidade. Logo, tem como base a família, pois é nesse contexto que surgem as primeiras noções de valor, respeito e incentivo, podendo ser influenciada, por exemplo, negativamente por críticas recebidas.

Outras situações prejudiciais aos níveis de autoestima estão relacionadas a estética e ao baixo condicionamento físico. A diminuição de atividade física, que traz consigo o aumento do estresse e do sedentarismo, pode ser explicada pelas tendências da sociedade em diminuir as exigências de movimentos corporais devido ao avanço tecnológico (Nahas, 2006; 
Barros et al., 2015). Nesse sentido, de acordo com o último Censo realizado no Brasil, estima-se que apenas $40 \%$ da população brasileira pratique algum tipo de exercício físico ou esporte (IBGE, 2010).

No entanto, pesquisadores apontam para a crescente busca da população por modalidades esportivas e práticas de exercício físico visando melhorar a qualidade de vida (Romero et al., 2017). Em contrapartida, a influência causada pelo investimento da mídia, a indústria de beleza e as redes sociais sobre esta geração tem estimulado o culto ao corpo e a busca pela beleza, ocasionando uma supervalorização da magreza e do fitness, principalmente em mulheres (Palhares, 2019). Com isso, é possível afirmar que a sociedade contemporânea se preocupa, sobremaneira, muito com a imagem corporal e estética.

Pessoas insatisfeitas com a sua imagem corporal têm dificuldades nas relações interpessoais, podendo ocasionar o aparecimento de quadros depressivos. Para tanto, a prática de exercício físico surge como uma forma de modelagem corporal, proporcionando melhorias de autoimagem, autoconceito e autoconfiança (Mazo et al., 2012; Coutinho, Tomazeti \& Acosta, 2013). Enquanto modalidade popular de prática de exercício físico, a musculação auxilia na prevenção e tratamento de doenças crônicas não transmissíveis e no ganho de massa magra, além de garantir benefícios que vão além do nível de condicionamento físico e nível emocional, capazes de inferir respostas fisiológicas positivas no organismo (Tavares Junior \& Planche, 2016).

Ainda, cabe mencionar que autores como Barros et al. (2015) e Brito et al. (2021) apontam o aumento significativo de praticantes na modalidade. Dessarte, considerando a relação entre os níveis de autoestima e a prática de exercício físico, assim como a influência da autoestima nas atividades diárias e os benefícios do treinamento resistido para a saúde física, mental e social do indivíduo, se fez oportuno reforçar a literatura por meio desse estudo que teve por objetivo avaliar e comparar os níveis de autoestima de praticantes masculinos e femininos de musculação.

\section{Metodologia}

\subsection{Modelo de pesquisa}

Trata-se de pesquisa de abordagem quantitativa (Zanella, 2013), de delineamento transversal (Thomas, Nelson \& Silverman, 2012) e de caráter descritivo e comparativo (Gil, 2008).

\subsection{Amostra}

De amostragem não-probabilística e do tipo intencional (Marconi \& Lakatos, 2003), a amostra foi composta por voluntários os quais se teve acesso, todos praticantes de musculação. Compuseram a amostra 120 praticantes de musculação, sendo 60 do sexo feminino (F) e 60 do sexo masculino (M), com média de idade de 25 anos em ambos os grupos $(\mathrm{F}=25,33 \pm 3,68 ; \mathrm{M}=25,93 \pm 3,85)$. Foram incluídos indivíduos praticantes de musculação há, no mínimo, 12 meses, cuja prática se configurasse em: no mínimo, três vezes na semana e de forma ininterrupta. Foram excluídos indivíduos que declararam ter distúrbios psicológicos e/ou que fizessem uso de medicamentos que pudessem vir a influenciar as respostas ao instrumento.

\subsection{Aspectos éticos}

O estudo seguiu as normas éticas previstas na Resolução no 466/2012 do Conselho Nacional de Saúde quanto à assinatura dos Termos de Consentimento Livre e Esclarecido, sendo informado a todos sobre seu anonimato. Esse estudo faz parte da linha de pesquisa de lombalgia e atividade física do grupo de pesquisa Biodinâmica do Desempenho, Exercício e Saúde (BIODESA), submetida ao Comitê de Ética em Pesquisa da Universidade Federal Rural do Rio de Janeiro (COMEPUFRRJ), sob o parecer de n $1318 / 19$. 


\subsection{Coleta de dados}

Para a coleta de dados, recorreu-se a Escala de Autoestima de Rosenberg (EAR), sendo este um questionário de autorresposta, composta por 10 perguntas, sendo cinco (5) referentes à autoimagem ou ao autovalor positivo e cinco (5) à autoimagem negativa ou autodepreciação (Rosenberg, 1989). As opções de resposta estão organizadas em uma escala tipo likert de quatro (4) pontos, variando entre: $0=$ concordo plenamente, $1=$ concordo, $2=$ discordo, $3=$ discordo plenamente; cujos valores dos itens positivos são invertidos e somados aos valores dos itens negativos, para obter-se a pontuação final. A pontuação pode variar de zero (0) a 30 pontos, na qual zero (0) representa o melhor estado e 30 o pior estado de autoestima. A partir disso, o indivíduo é classificado em dois níveis: alta autoestima ou baixa autoestima (Rosenberg, 1989; Heatherton \& Wyland, 2003).

A coleta de dados ocorreu nos meses de setembro e outubro de 2019, antes dos decretos de lockdown e das medidas sanitárias em resposta a pandemia da Covid-19 serem implementadas.

\subsection{Análise Estatística}

O tratamento dos dados foi realizado estatisticamente no software SPSS 20.0. A caracterização dos níveis de autoestima foi realizada a partir do cálculo de medidas de tendência central e dispersão, isto é, foram apresentadas as médias e desvio padrão dos resultados por pergunta em cada grupo. No que diz respeito as comparações, o teste de normalidade de Shapiro Wilk determinou que os dados são não paramétricos. Desta forma, foi aplicado o teste de Mann Whitney com nível de significância de $\mathrm{p} \leq 0,05$ (Vieira, 2016).

\section{Resultados}

A seguir, os resultados ilustrados na Tabela 1 apresentam as médias e desvio padrão obtidos em função das respostas por perguntas na Escala recorrida, bem como o p valor das comparações realizadas em função do sexo. De maneira geral, o grupo do sexo feminino indicou média de 2,57 $\pm 0,61$ e o grupo do sexo masculino $2,57 \pm 0,78$, demonstrando similaridade em função do nível de autoestima nos grupos investigados. As comparações não indicaram diferença com significância estatística em relação a autoestima.

Tabela 1. Resultados em função das respostas por questão na EAR.

\begin{tabular}{lcc}
\hline QUESTÕES & $\begin{array}{c}\text { MULHERES } \\
\text { Md } \pm \text { DP }\end{array}$ & $\begin{array}{c}\text { HOMENS } \\
\text { Md } \pm \text { DP }\end{array}$ \\
\hline Q1 & $3,03 \pm 0,76$ & $3,33 \pm 0,61$ \\
Q2 & $2,03 \pm 0,81$ & $1,50 \pm 0,63$ \\
Q3 & $3,27 \pm 0,52$ & $3,43 \pm 0,50$ \\
Q4 & $3,00 \pm 0,69$ & $2,93 \pm 0,94$ \\
Q5 & $2,13 \pm 1,01$ & $1,90 \pm 0,66$ \\
Q6 & $2,10 \pm 0,84$ & $1,87 \pm 0,86$ \\
Q7 & $3,27 \pm 0,69$ & $3,47 \pm 0,57$ \\
Q8 & $2,13 \pm 0,82$ & $2,30 \pm 0,92$ \\
Q9 & $1,67 \pm 0,71$ & $1,57 \pm 0,73$ \\
Q10 & $3,13 \pm 0,78$ & $3,40 \pm 0,50$ \\
\hline GERAL & $2,57 \pm 0,61$ & $2,57 \pm 0,78$ \\
\hline MÉDIA DA SOMA & $25,77 \pm 2,62$ & $25,70 \pm 2,59$ \\
\hline
\end{tabular}

Legenda: Md=Média; DP=Desvio Padrão; Q=Questão. Fonte: Autores. Dados da pesquisa. 
Em função das respostas por pergunta, os dados se equivalem entre homens e mulheres, especialmente nas questões: Q1 (Eu sito que sou uma pessoa de valor, no mínimo, tanto quanto as outras pessoas); Q3 (Levando tudo em conta, eu penso que eu sou um fracasso); Q4 (Eu acho que sou capaz de fazer as coisas tão bem quanto a maioria das pessoas); Q7 (No conjunto, eu estou satisfeito comigo); e Q10 (Às vezes eu acho que não presto para nada). Isto é, todos concordaram.

Ainda de forma equivalente, nas questões: Q5 (Eu acho que eu não tenho muito do que me orgulhar); Q6 (Eu tenho uma atitude positiva com relação a mim mesmo); e Q8 (Eu gostaria de poder ter mais respeito por mim mesmo) todos discordaram. Na Q9 (Às vezes eu me sinto inútil), ambos os grupos discordaram totalmente. Diferente disso, na Q2 (Eu acho que eu tenho várias boas qualidades), os homens apenas discordaram, mas as mulheres discordaram totalmente.

Em cinco questões da EAR, o grupo do sexo masculino obteve percentual acima de 50\% de satisfação. O mesmo aconteceu entre as mulheres. Nesse contexto, cabe mencionar que a classificação de autoestima é definida como: alta (satisfatória) - escore maior que 30 pontos; média - escore entre 20 e 30 pontos; e baixa (insatisfatória) - escore menor que 20 pontos (Rosenberg, 1989). De acordo com a classificação proposta para a Escala recorrida, ambos os grupos possuem autoestima média.

\section{Discussão}

Rememorando, os resultados encontrados nesse estudo foram: similaridade em função do nível de autoestima nos grupos investigados, sendo esta classificada, a partir dos escores alcançados, como autoestima média. No que diz respeito as comparações, não se evidenciou diferença com significância estatística. Devido ao fato de a autoestima estar relacionada diretamente com a qualidade de vida, diversos autores se propuseram a avaliar a autoestima em diferentes populações, como adolescentes (Melo et al., 2021), idosos institucionalizados (David et al., 2020) e pacientes renais crônicos (Silva et al., 2021). Além disso, alguns estudos evidenciaram relação significativa entre a baixa autoestima e a prevalência de transtornos mentais comuns (Preto et al., 2020) e esquizofrenia (Aparecido \& Silva, 2020).

Objetivando verificar os motivos para adesão à prática de musculação em uma academia no Município de Parintins/AM-BR, 35 adultos ( $\mathrm{M}=16 ; \mathrm{F}=19)$ foram investigados, utilizando um questionário composto de perguntas abertas e fechadas. Cabe mencionar que a maioria dos indivíduos $(68,6 \%)$ indicaram praticar musculação há mais de um ano e todos (100\%) com frequência semanal maior do que três (3) vezes na semana. Em função dos resultados, um dos principais motivos para prática de musculação foi a melhora da autoestima (97,1\%), citada por 34 dos 35 participantes da pesquisa (Barros et al., 2015).

No mesmo sentido, Liz e Andrade (2016) entrevistaram 21 indivíduos, de ambos os sexos, com o intuito de investigar os motivos de adesão ( $n=13 ; M=6$ e H=7) e desistência ( $n=8 ; M=5$ e H=3) da prática de musculação. Dentre os resultados, a autoestima não foi citada como motivo à prática, no entanto foi mencionada por quatro participantes, sendo relacionada aos benefícios psicológicos da musculação (melhora a autoestima), ainda que considerada menos frequente no grupo investigado, levando em conta o seu número de aparecimento.

Ainda, ao realizar uma pesquisa bibliográfica, com o intuito de relatar os aspectos à adesão e motivação à prática de musculação nas academias do Brasil, Brito et al. (2021) pontuaram que um dos principais fatores/motivação é o aumento da autoestima. Cabe pontuar a possibilidade de a prática de atividade física e/ou esportiva regular influenciarem nos níveis de autoestima, de forma a elevá-los, embora a prática não seja o único fator capaz de influenciar esses níveis (Aguiar et al., 2019).

Dessa forma, a autoestima é um dos motivos citados para a prática de musculação (Barros et al., 2015; Liz \& Andrade, 2016; Brito et al., 2021), sendo possível elevar seus níveis mediante a prática regular de atividades físicas e/ou esportivas (Aguiar et al., 2019). Nesse sentido, é relevante desenvolver estudos como este, bem como dialogar com outros achados na literatura sobre a temática (Tafarello, Nascimento Júnior \& Oliveira, 2015; França, 2017; Silva et al., 2019). 
Utilizando também a EAR, buscou-se analisar a qualidade de vida e autoestima de 30 mulheres, do Município de Maringá/PR-BR, sendo 15 praticantes de musculação e 15 praticantes de ginástica. Ambos os grupos obtiveram quase que escore máximo na EAR e, por isso, a autoestima foi classificada como boa. Dialogando com os achados deste estudo, não foram encontradas diferenças significativas entre os grupos em relação a autoestima (Tafarello, Nascimento Júnior \& Oliveira, 2015).

Tendo como objetivo analisar o nível de autoestima, percepção e satisfação com a imagem corporal, França (2017) investigou 103 homens praticantes de diferentes tipos de treinamento contra a resistência (musculação e crossfit; treinamento funcional e pilates), sendo 53 homens praticantes de musculação e crossfit. Para avaliar a autoestima utilizou-se a EAR. Diferente dos resultados aqui apresentados, na comparação entre grupos a autoestima foi classificada insatisfatória (42,6\%) para os praticantes de musculação e crossfit, podendo esta ser influenciada, positiva ou negativamente, pelo "[...] tipo de treinamento e seu consequente nível de muscularidade [...]" (p. 27).

Os resultados encontrados por Silva et al. (2019) dialogam com os achados nesse estudo. Os autores buscaram investigar a motivação, o bem-estar e a autoestima entre 31 indivíduos, praticantes de três modalidades, sendo uma delas a musculação $(n=21)$. Para tal, recorreu-se a três instrumentos, sendo um deles a EAR. Os resultados apontaram escore moderado de autoestima e, ainda, essa foi a única variável que não apresentou diferenças significativas entre os grupos (treinamento funcional e musculação com/sem personal trainer).

\section{Considerações Finais}

Ao avaliar e comparar os níveis de autoestima de praticantes masculinos e femininos de musculação, os resultados obtidos indicaram que, independente do sexo, com o tempo de prática de musculação equivalentes, os praticantes possuem autoestima semelhante, classificada como média. Nesse contexto, atribui-se os benefícios na autoestima como consequência do exercício da musculação.

No que se refere as comparações, não houve diferença com significância estatística em relação a autoestima. Esperase que os achados aqui apresentados contribuam para ampliar a compreensão acerca dos fatores psicológicos e sua relação com a prática regular de atividade física, bem como para auxiliar futuras intervenções dos profissionais que atuam nessa área.

Por fim, novos estudos devem ser realizados visando ampliar a compreensão do fenômeno estudado com, por exemplo, maior número de participantes e controle de outras variáveis como nutrição, vínculo empregatício e horas de sono.

\section{Referências}

Aguiar, D. M. M., Azevedo, E. D., Borges, M. A. O., Lima, B. \& Lima, V. P. (2019). Autoestima entre alunos iniciantes e concluintes do Curso de Licenciatura em Educação Física. Conhecimento Interativo, 13(1), 194-201. http://app.fiepr.org.br/revistacientifica/index.php/conhecime ntointerativo/article/view/318/343

Aparecido, G. A., \& Silva, D. A. da. (2020). Self-esteem assessment in people with schizophrenia. Research, Society and Development, 9(8), e821986449. https://doi.org/10.33448/rsd-v9i8.6449

Barros, G. R., Castellano, S. M., Santos, S. F. S. \& Paulo, T. (2015). Motivos para a prática de musculação entre adultos jovens de uma academia no Município de Parintins/AM. Revista Acta Brasileira do Movimento Humano, 5(1), 66-75. http://www.periodicos.ulbra.br/index.php/actabrasi leira/article/view/2852/2119

Brito, G. C., Bernaldino, E. S., Almeida, E. A., Santos, L. J. A. M., Antunez, B. F., Silva, O. G., Rodrigues, M. A. M., Oliveira, J. R. L., Oliveira, H. F. R., Pereira, A. A., Lima, B. N., Fileni, C. H. P., Martins, G. C., Camargo, L. B., Carvalho, A. F., Passos, R. P., Vilela Junior, G. B. \& Silio, L. F. (2021). Adesão a prática de musculação nas academias do Brasil. Revista CPAQV - Centro de Pesquisas Avançadas em Qualidade de Vida, 13(1), 1-7. https://dx.doi.org/10.36692/v13n1-2

Coutinho, R. X., Tomazeti, R. V. \& Acosta, M. A. F. (2013). Representação de corpo na velhice: o corpo real versus o corpo social. Revista Kairós Gerontologia, 16(4), 215-236. https://doi.org/10.23925/2176-901X.2013v16i4p215-236

David, C. S. de, Cerezer, J. P., Moura, L. V., Flores, M. T. F. D., Machado, V. K., Rodrigues, R. F. de L., \& Carlesso, J. P. P. (2020). The self-esteem of elderly women in a support association: experience report. Research, Society and Development, 9(3), e57932364. https://doi.org/10.33448/rsd-v9i3.2364 
França, M. C. (2017). Nível de autoestima e percepção da imagem corporal em homens praticantes de diferentes treinamentos contra a resistência. Trabalho de Conclusão de Curso (Bacharelado em Educação Física) - Universidade Federal do Rio Grande do Norte, Natal/RN.

GIL, A. C. (2008). Métodos e Técnicas de Pesquisa Social. (6a ed.), Editora Atlas S.A.

Gobitta, M. \& Guzzo, R. S. L. (2002). Estudo inicial do inventário de Auto-Estima (SEI): Forma A. Psicologia: Reflexão e Crítica, 15(1), 143-150. https://doi.org/10.1590/S0102-79722002000100016

Heatherton, T. F. \& Wyland, C. L. (2003). Assessing self-esteem. Assessing positive psychology. Todd.

IBGE. Instituto Brasileiro de Geografia e Estatística. (2010). Características gerais da população, religião e pessoas com deficiência, segundo a situação do domicílio e grupos de idade. https://biblioteca.ibge.gov.br/visualizacao/periodicos/94/cd_2010_religiao_deficiencia.pdf

Liz, C. M. \& Andrade, A. (2016). Análise qualitativa dos motivos de adesão e desistência da musculação em academias. Revista Brasileira de Ciências do Esporte, 38(3), 267-274. https://doi.org/10.1016/j.rbce.2015.11.005

Marconi, M. A. \& Lakatos, E. M. (2003). Fundamentos de metodologia científica. (5a ed.), Editora Atlas S. A.

Mazo, G. Z., Krug, R. R., Virtuoso, J. F., Streit, I. A. \& Benetti, M. Z. (2012). Autoestima e depressão em idosos praticantes de exercícios físicos. Revista Kinesis, 30(1), 188-199. http://dx.doi.org/10.5902/010283085724

Melo, L. B. C. de, Barbosa, I. T. de L., Silva, P. S. D. da, \& Silva, J. G. M. da. (2021). Self-esteem in adolescent students from two state schools in the City of Nazaré da Mata - PE. Research, Society and Development, 10(5), e44410514988. https://doi.org/10.33448/rsd-v10i5.14988

Morales, M. \& González, A. (2014). Resiliencia-Autoestima-Bienestar psicológico y Capacidad intelectual de estudiantes de cuarto medio de buen rendimiento de liceos vulnerables. Estudios Pedagógicos, Valdivia, 40(1), 215-228. http://dx.doi.org/10.4067/S0718-07052014000100013

Nahas, M. V. (2006). Atividade Física, Saúde e Qualidade de Vida: conceitos e sugestões para um estilo de vida ativo. (4a ed.), Midiograf.

Oliveira, S. M. L. P. (2001). O impacto do exercício físico na auto-estima, investimento corporal e sentimentos de auto-eficácia em estudantes universitárias: estudo exploratório. Psicologia, o Portal dos Psicólogos. https://www.psicologia.pt/artigos/textos/TL0010.PDF

Palhares, T. (2019). Bem-estar mental e emocional na atualidade: Saúde além do corpo. Editora Conquista.

Preto, V. A., Fernandes, J.M., Silva, L. P. da, Reis, J. O. L. dos, Sousa, B. de O. P., Pereira, S. de S., Sailer, G.C., \& Cardoso, L. (2020). Common Mental Disorders, Stress and Self esteem in university students in the health field in the last year. Research, Society and Development, $9(8)$, e844986362. https://doi.org/10.33448/rsd-v9i8.6362

Romero, B. F., Castanho, G. K. F., Chiminazzo, J. G. C., Barreira, J. \& Fernandes, P. T. (2017). Motivação e atividade física: os projetos de extensão na universidade. Pensar a Prática, Goiânia, 20(1), 73-87. https://doi.org/10.5216/rpp.v20i1.44134

Rosenberg, M. (1989). Society and the adolescent self-image. Revised edition. Middletown, Morris. 148-168. https://psycnet.apa.org/record/1990-98431-000

Salmivalli, C., Kaukiainen, A. O., Kaistaniemi, L. \& Lagerspetz, K. M. J. (1999). Self-Evaluated Self-Esteem, Peer-Evaluated Self-Esteem, and Defensive Egotism as Predictors of Adolescents' Participation in Bullying Situations. Personality and Social Psychology Bulletin, 25(10), 1268-1278. https://journals.sagepub.com/doi/10.1177/0146167299258008

Schultheisz, T. S. V. \& Aprile, M. R. (2013). Autoestima, conceitos correlatos e avaliação. Revista Equilíbrio Corporal e Saúde, 5(1), 36-48. https://doi.org/10.17921/2176-9524.2013v5n1p\%25p

Silva, T. C., Freire, G. L. M., Morais, O. S. G. \& Nascimento Junior, J. R. A. (2019). Motivação, bem-estar e autoestima de praticantes de diferentes modalidades de exercício físico. Saúde e Pesquisa, Maringá, PR, 12(2), 359-366. https://doi.org/10.17765/2176-9206.2019v12n2p359-366

Silva, L. J. de A., Santos, S. V. M. dos., Dázio, E. M. R., Soares, M. I., Nogueira, D. A., \& Terra, F. de S. (2021). Anxiety and self-esteem in chronic kidney patients submitted to hemodialytic treatment. Research, Society and Development, 10(10), e46101018406. https://doi.org/10.33448/rsd-v10i10.18406

Tafarello, R., Nascimento Júnior, A. \& Oliveira, D. V. (2015). Qualidade de vida e autoestima de mulheres praticantes de musculação e ginástica em academia. Cinergis, Santa Cruz do Sul, 16(4), 249-254. http://dx.doi.org/10.17058/cinergis.v16i5.6667

Tavares Junior, A. C. \& Planche, T. C. (2016). Motivos de adesão de mulheres a prática de exercícios físicos em academias. Revista Equilíbrio Corporal e Saúde, 8(1), 28-32. https://doi.org/10.17921/2176-9524.2016v8n1p28-32

Thomas, J. R., Nelson, J. K. \& Silverman, S. J. (2012). Métodos de Pesquisa em Atividade Física. (6a ed.), Artmed.

Vasconcelos, H. S. (2017). Autoestima, autoimagem e constituição da identidade: um estudo com graduandos de Psicologia. Revista Psicologia, Diversidade e Saúde, 6(3), 195-206. https://doi.org/10.17267/2317-3394rpds.v6i3.1565

Vieira, S. (2016). Introdução à Bioestatística. (5a ed.), Guanabara Koogan.

Zanella, L. C. H. (2013). Metodologia de Pesquisa. (2a ed.), Departamento de Ciências da Administração, UFSC. 